\title{
IS INTERPROFESSIONAL PRACTICE (IPP) FOCUSED ON MEDICATION SAFETY FEASIBLE IN INDONESIA? A QUALITATIVE STUDY
}

\author{
APAKAH KERJASAMA ANTAR PROFESI UNTUK KEAMANAN PEMAKAIAN OBAT DAPAT \\ DITERAPKAN DI INDONESIA? SEBUAH STUDI KUALITATIF
}

\author{
Desak Ketut Ernawati ${ }^{1}$, Ya Ping Lee ${ }^{2}$, Bruce Sunderland ${ }^{2}$ Jeff Hughes ${ }^{2}$ \\ 1) School of Medicine, Udayana University, Bali Indonesia \\ 2) School of Pharmacy, Curtin University, Perth, Western Australia
}

\section{ABSTRACT}

\begin{abstract}
This paper explores facilitators for and barriers to the implementation of IPP focusing on medication safety in a public hospital in Bali, Indonesia. Qualitative methods involved interviews with stakeholders from a university and a hospital and focus group discussions with healthcare professionals in the hospital. Semi-structured questions were developed as a guide for the interviews and discussions. All interviews and discussions were recorded. The six steps of Braun and Clarke's thematic analysis methodology were implemented in determining the themes. The Consolidated Criteria for Reporting Qualitative Research (COREQ) checklist was employed in reporting of findings. Participants indicated that support from the government and perceived benefits of IPP were facilitators for IPP. However, the participants mostly mentioned the barriers of IPP including lack of competencies for IPP and lack of understanding of the role of other healthcare professionals as barriers to the implementation of IPP. This showed that these were the barriers identified to the implementation of IPP in the study hospital. Despite the fact that participants were supportive of IPP, the participants identified some barriers to the implementation of IPP in the study hospital. The implementation requires support of the government, professional organisations, and stakeholders at the university and hospital levels.
\end{abstract}

Keywords : Interprofessional practice, medication safety, Indonesia, qualitative study

\section{INTRODUCTION}

Developed and developing countries have no significant differences in terms of the perceived benefits of interprofessional education for teaching, learning, practice and policy (Rodger and Hoffman, 2010). Although some similarities of the benefits were found in both developed and developing countries, some differences were identified (Mickan et al, 2010). A governance model may facilitate shared responsibility amongst healthcare professionals in developed countries, meanwhile, the hierarchical model of healthcare service delivery is considered as one of the barriers in developing countries. Mickan et al. (2010) suggested that collaboration amongst healthcare professionals is essential in complex healthcare service delivery to ensure a safe and quality service. However, they stated that lack of access to medical records for healthcare professionals in developing countries may restrict interprofessional practice (IPP).

\section{Corresponding}

\section{Desak Ketut Ernawati}

School of Medicine, Udayana University, Bali

Email : ketuternawati@unud.ac.id
A framework of interprofessional education (IPE) for collaborative patient centred practice (IECPCP) is one of the methods to assess the feasibility of the implementation of IPP aimed at facilitating collaborative practice in patient care (D'Amour and Oandasan, 2005). This framework has three levels (i.e. Micro, Meso and Macro). The framework was selected because it takes into consideration factors at the individual and organisation levels which may influence collaborative practice. The Micro level focuses on the needs of the patient and interactional factors between individuals and healthcare professionals, as well as with the healthcare organisation. The Meso level emphasises organisational factors such as governance and healthcare service structure. Meanwhile, the Macro level is influenced by professional systems, government policies, and social and cultural values of healthcare professionals. In the current Indonesian practice model, little is known regarding how IPP is adopted in healthcare service delivery. This study aimed to assess the feasibility of implementing IPP, focusing on medication safety in one hospital in Bali, Indonesia. Medication safety was selected as the topic for the present study because communication and 
accurate information transfer are crucial amongst the different healthcare professionals (i.e. physician, nurse, and pharmacist) who are involved in the process of medication delivery. This study aimed to explore the feasibility of the IPP implementation focused on medication safety in Indonesian practice; identifying facilitators and barriers for stakeholders and healthcare professionals.

\section{METHODS}

The primary investigator performed semi-structured interviews and focus group discussions (FGDs). Three domains of the Consolidated Criteria for Reporting Qualitative Research (COREQ) checklist was employed in reporting of the findings (Tong, et al., 2007). The study approval was granted by the Curtin Human Research Ethic Committee (HREC) and Ethics Committee of the study university. Informed consent was obtained for each of the interviews and responding to the invitation to attend the FGDs was considered as giving consent.

The first domain of COREQ consists of personal characteristics and relationship with participants. The participants of the present study knew the primary investigator (DKE) prior to the study commencement and understood the aims of her research as part of a $\mathrm{PhD}$ study. The primary investigator (DKE) conducted the interviews. Meanwhile, FGDs were facilitated by an independent facilitator.

The second domain of COREQ is study design which encompasses participant selection, setting, theoretical framework and data collection. Participants in the interviews were purposefully recruited and comprised the Heads of the Medical, Nursing, and Pharmacy Departments from a public university, together with the Director of the Hospital and Head of the Pharmacy Department in a public hospital in Bali, Indonesia. The interviews were conducted in the interviewees' offices. Meanwhile, participants in FGDs were selected randomly from lists of practising physicians, nurses and pharmacists from the same public hospital in Bali, Indonesia. The FGDs were conducted in the study hospital. Semi-structured questions were developed as a guide for the interviews and FGDs. The questions sought to assess participants' support towards IPP, as well as potential facilitators and barriers to its implementation, based on the current practice of healthcare service delivery. This was to provide consistency in the manner in which the primary investigator and the facilitator conducted the interviews and FGDs, while helping ensure that the interviews and FGDs stayed on topic.

All interviews and discussions used audio recording to ensure accuracy of transcription. The six steps of Braun and Clarke's thematic analysis methodology were implemented in determining the themes (Braun and Clarke, 2006). The primary investigator was familiar with the transcripts of interviews and FGDs because she translated and transcribed the interviews herself. Coded themes were grouped together for each interview as part of the thematic analysis process.

The third domain of COREQ consists of data analysis and reporting. $\mathrm{NVivo}^{\mathrm{TM}}$ data management was employed. Interviewees reviewed the transcription to check for correctness while the facilitator reviewed the FGDs' transcriptions for checking. The themes were data driven. Frequencies of themes arising from interviews and FGDs were also determined to obtain an understanding of theme distributions and the level of their importance with respect to the research questions.

\section{RESULTS AND DISCUSSION}

All of the interviewees were male. There were seven medical doctors (physicians), six nurses and eight pharmacists in the hospital participated in the FGDs. Initially, there were one physician, one pharmacist and two nurses were unable to attend the FGDs because of their commitments at the hospital. However, the primary investigator could only recruit one pharmacist to replace those who unable to come. The majority of participants in the FGDs were female and had been working in the hospital for between one to 25 years. The interviews and discussions lasted approximately one hour. Translation and transcription were 
conducted by the primary investigator within 48 hours of the interviews and FGDs to ensure important notes taken during interviews/FGDs were not lost.

In general, the Heads of Departments from the university and hospital as well as participants in FGDs, were supportive towards IPP. Support of the Heads of Department can be seen in the following extracts:

"I absolutely agree at level 10 [10 of being the highest level of agreement] because, we always think about the patients" (Head of Hospital 2)

Support extracted from FGDs and the participants' group responses are illustrated in the following:

Facilitator of FGD: "Do you agree to work with other professions to provide healthcare service?" All participants in Nurse Group said yes and laugh.

Facilitator of FGD: "...how many ... agree to work with other professions to ensure the safe use of medication? "All participants in Pharmacy Group raised their hands. P1-PG: "It should be everyone as long as we keep our knowledge updated..."

Facilitator of FGD: "Do you agree to work in a team with other team members in providing patient care?" Participants 1- Physician Group: "Yes, we do. Happy about it, we like to communicate. We'd love to call them or text them (laughing)..."

Table I shows themes identified which related to facilitators for and barriers to the implementation of IPP in the study hospital from the interviews and FGDs. As can be seen in Table I, participants in the study discussed more frequently the barriers rather than the facilitators for implementing IPP in the study hospital.

To the best of our knowledge, this is the first Indonesian study aimed at identifying facilitators for and barriers to the implementation of IPP focused on medication safety in a hospital setting in Indonesia.

\section{Facilitators for IPP}

Participants in the present study indicated the Indonesian Government supported IPP with the adoption of the Joint Commission International (JCI) accreditation as part of the Indonesian Government policy is to ensure patient safety in healthcare service delivery. Participants of the present study also identified that IPP may potentially bring positive benefits not only to minimise the potential risk of medication misadventure, but also reducing blame to certain healthcare professionals when the misadventure occurred. Expectation towards the role of healthcare providers was mentioned by the participants. They claimed that other healthcare professionals (e.g. nurses and physicians) thought the role of pharmacists on the wards was mainly about logistics (i.e. medicine supply) and in reducing the workload involved in drug distribution. The stakeholder's expected the interaction between nurses and pharmacists on the wards was more focussed on medication advice. This suggested that the stakeholder supports IPP amongst healthcare professionals.

Participants stated that there was limited interaction between healthcare providers. Participants identified that physicians mostly work with nurses but had very limited interaction with pharmacists. The interviewed pharmacist described most pharmacists' interactions were with nurses and limited interaction with physicians. Discussion with the Pharmacist Group also revealed different opinions on the level of communication between pharmacists and physicians in the hospital. It appeared that the level of relationship was dependent upon the wards and place of work. Although the level of communication amongst physicians and pharmacists was thought to be poor, potential interaction may occur when the medication prescribed was outside the hospital formularies or the prescription order was unclear due to poor hand writing necessitating pharmacist intervention.

\section{Barriers to IPP}

The barriers identified were lack of competencies in IPP, lack of understanding of the role of healthcare professionals, superiority, no legislation from the government on teamwork, and limited staff. Although participants agreed competencies in IPE are important, participants indicated that health care professionals lacked these competencies. Knowledge competency was not only within one's own profession but also knowledge of the 
Table I. Themes identified as facilitators for and barriers to the implementation of IPP focused on medication safety

\begin{tabular}{|c|c|c|c|c|c|c|c|c|c|}
\hline $\begin{array}{c}\text { Facilitators/ } \\
\text { Barriers }\end{array}$ & Themes & HU1 & HU2 & HU3 & HH1 & HH2 & MG & NG & PG \\
\hline \multirow[t]{4}{*}{ Facilitators } & Benefits of IPP & ++ & + & + & + & ++ & + & + & + \\
\hline & $\begin{array}{l}\text { Expectation towards the role } \\
\text { of others }\end{array}$ & + & + & + & + & + & + & + & + \\
\hline & $\begin{array}{l}\text { Interaction between } \\
\text { healthcare professionals }\end{array}$ & + & + & + & + & + & + & ++ & + \\
\hline & Support of IPP & + & + & ++ & ++ & + & + & + & + \\
\hline \multirow[t]{5}{*}{ Barriers } & Lack of competencies in IPP & ++ & +++ & +++ & ++ & +++ & ++ & ++ & +++ \\
\hline & $\begin{array}{l}\text { Lack of understanding of the } \\
\text { role of healthcare professional }\end{array}$ & ++ & +++ & + & + & ++ & +++ & +++ & ++ \\
\hline & Superiority & + & + & + & + & + & + & + & + \\
\hline & $\begin{array}{l}\text { No legislation from the } \\
\text { government on teamwork }\end{array}$ & + & + & ++ & ++ & + & + & + & + \\
\hline & Limited staff & + & + & ++ & ++ & + & + & + & + \\
\hline
\end{tabular}

Notes: +++: themes identified more than 50 times; ++: themes identified 25-50 times; +: themes identified less than 25 times; HU: Heads of Department in the university; HH; Heads in the hospital, MG: Medical Doctor Group; NG; Nurse Group; PG: Pharmacist Group

roles of other healthcare professionals. Communication and teamwork skills emerged as skills or competencies in IPP in the present study. These skills included what and how to deliver a message to other health care professionals. Failure of clear delivery of the message might cause ineffective communication. However, one of the Heads interviewed highlighted that currently there was a lack of role models amongst practitioners with good communication skills in practice. Participants also stated that teamwork is essential in working inter-professionally in healthcare service delivery.

Trust and respect were identified as attitude sub-themes. Participants suggested that trust of other healthcare professionals and patients are essential in IPP. For example, if physicians doubted the pharmacists' competency to dispense medication for paediatric patients, this may reduce the physicians' trust in working with the pharmacists in a team. Respect was the most common attitude mentioned during interviews.
Respect of other healthcare professionals is essential in teamwork. The belief of the role of other healthcare professionals as a physicians' helper was identified in the present study. This may reflect the culture of healthcare service delivery in Indonesia where the physician assumes the highest position within the healthcare hierarchy. This was also identified in another theme as a barrier to IPP which was superiority. As per dictionary definitions (Oxford, 2015) "ego" is defined as a person's self-importance (in this case the importance of certain professions within the healthcare delivery). "Hierarchy" is defined as the level of arrangement based on the importance in an organisation. "Superior" relates to higher status or power (in this case in health service delivery). Based on these definitions, ego, hierarchy, and superiority were considered as one theme of superiority. Social, relational and the structure of an organisation may result in communication failures in healthcare service delivery (Sutcliffe et al., 2004). 
Stakeholders in the university suggested that there is no legislation to cover working and sharing the responsibility within a team. At the study hospital, there was no explicit legislation on teamwork (collaboration) amongst healthcare professionals. The lack of adequate numbers of healthcare professionals was also mentioned as one cause of sub-optimal healthcare services which could be one of barriers to the implementation of IPP.

\section{Feasibility of IPP implementation based of IECPCP}

At the Micro Level, this study identified that healthcare professionals realised the importance of IPP in ensuring medication safety at the study hospital. Participants in interviews, as well as FGDs, discussed the benefits not only to the patient but also to healthcare professionals. In this regard, the healthcare professionals had identified that IPP may bring about positive benefits to the staff. If the staff perceived gaining advantages from IPP, this may also positively influence organisation performance in patient care. Ginsburg and Tregunno (2005) stated that organisational culture (i.e. values, beliefs, and attitudes) and climate (i.e. policies, procedures, and staff's belief in their organisation) have a strong influence on the level of successfulness of organisational performance in patient care. Thus, staff's positive behaviour is an important factor if IPP is to occur. The results of the present study have indicated that healthcare professionals were supportive towards IPP. This showed that support at the Micro level (healthcare professionals) of the framework of IECPCP was identified.

This study identified knowledge, skills and attitudes were competencies required in IPP. The knowledge competency included competency within one's own profession, as well as understanding of the roles of other healthcare professionals (WHO, 2010). Skills competency involved ability to communicate effectively amongst healthcare professionals as well as with the patients. Suter et al (2009) suggested that communication skills are an important competency of healthcare providers in IPP. The skills involved using the appropriate language and ability to negotiate with others. This means that healthcare professionals are required to learn dual identities - their own professional identity as well as an interprofessional identity (Khalili et al, 2013). Further, attitudes identified were also related to respect towards others (Baker, Gustafson and Beaubien, 2003).

Despite the fact that the competencies required were identified, participants in this study indicated that healthcare professionals had a lack of knowledge of the roles of other healthcare professionals. Understanding the role of healthcare professionals and communication skills are essential in collaborative practice (Suter et al., 2009). This study also indicated the sense of superiority amongst medical practitioners potentially may lead to limited interaction between healthcare professionals. The sense of superiority in healthcare service delivery found in the present study may result from a lack of understanding of the role of other healthcare professionals. This lack of understanding was identified as a major barrier for the implementation of IPP in the study hospital. This perceived superiority may also be one of the barriers to the implementation of IPP at the Micro level. The sense of superiority could be influenced by strong professional socialisation amongst physicians. Hafferty and Franks (1994) stated that professional identity in physicians was developed in an informal curriculum namely, a hidden curriculum. Similarly et al (2001) also described the informal stage as an important part of professional socialisation in graduates. Whitehead (2007) suggested that physicians' involvement in professional collaboration should be designed in a model which provided evidence of improving patient outcomes.

Stakeholders in the present study believed that IPP could potentially minimise the risk of medication misadventure. This suggested support at the Meso level of IECPCP existed in the study hospital. This support is essential for the implementation of IPP (Mickan et al., 2010). However, there was no standard protocol at the hospital to work in a team with other healthcare professionals. Although the study hospital 
adopted an international accreditation which made communication amongst healthcare professionals inevitable with the introduction of integrated notes, it is unknown whether the integrated notes had facilitated enhanced information exchange amongst healthcare professionals in performing their duties. The lack of standard protocols and the lack of facilities to work with other healthcare professionals have also been discussed in the literature as barriers to the implementation of IPP (Mickan et al., 2010; WHO, 2010)

At the Macro level, the participants indicated that the Indonesian Government supported interprofessional collaboration amongst healthcare professionals. However, participants felt that there was no clear legislation on teamwork in the provision of healthcare services in their present practice. Additionally, support from professional organisations towards IPP was not identified in the present study. Thus, further study may be required to identify support from the professional organisations. This is because support from the government and professional organisations is considered as significant drivers for the implementation of IPP (Mickan et al., 2010; WHO, 2010). The hierarchical model of healthcare delivery which may result from superiority identified in the present study mirrored barriers to implement IPP found in other developing countries (Mickan et al., 2010).

Although this study identified the implementation of IPP was feasible based on the IECPC framework in the study hospital, there were some potential sources of bias in this qualitative study. Bias may arise from both the participants as well as the researchers (Knox and Burkard, 2009). Bias from participants may be associated with their positive motivation to be involved in research. This may influence positive participants' responses towards the questions asked. This was beyond the control of the investigator. Meanwhile, bias from the researcher may result from the data collection and analysis process. Potential bias from the researcher was minimised by using a triangulation data collection method in which similar data were collected from interviews and
FGDs. This study has employed established checklists of analysing and reporting of qualitative data to improve the quality of qualitative research. However, this study was unable to meet all of the checklists. Another potential bias was this study was conducted in one university and one hospital in one province in Indonesia. Thus, generalisation of the findings should be made cautiously in other settings in the country.

\section{CONCLUSION}

This study found that the Indonesian Government and the stakeholders support IPP implementation. However, a lack of understanding of the roles of healthcare professionals, no clear guidelines of working collaboratively, superiority and limited staff numbers were identified as barriers to the implementation of IPP in the study hospital. The lack of understanding of the role of other healthcare professionals may emanate from the hierarchical model which exists currently in healthcare service delivery. The barriers identified, which are similar to those identified in other developing countries, will need to be addressed if IPP is to be implemented in the study hospital, and more broadly in Indonesia. This required support from the government, professional organisations, and stakeholders.

\section{ACKNOWLEDGEMENTS}

The investigators thank to stakeholders in the university and hospital as well as healthcare professionals who participated in the present study.

\section{REFERENCES}

Baker DP, Gustafson S, Beaubien J., 2003. Medical teamwork and patient safety: the evidence-based relation 2003 Center for Quality Improvement and Patient Safety: Agency for Healthcare Research and Quality (AHRQ).

Braun V, Clarke V., 2006. Using thematic analysis in psychology. Qual Res Psychol. 3:77-101.

D'Amour D, Oandasan I., 2005. Interprofessionality as the field of 
interprofessional practice and interprofessional education: an emerging concept. J Interprof Care. Supplement 1:820.

Ginsburg L, Tregunno D., 2005. New approaches to interprofessional education and collaborative practice: Lessons from the organizational change literature. J Interprof Care. Supplement 1:177-87.

Hafferty F, Franks R.,1994. The hidden curriculum, ethics teaching and the structure of medical education. Acad Med [abstract]. 69:861-71.

Khalili H, Orchard C, K H, Laschinger S, Farah R.,2013. An interprofessional socialization framework for developing an interprofessional identity among health professions students. Journal of Interprofessional Care. 27:448-53.

Knox S, Burkard AW.,2009 Qualitative research interviews. Psychother Res. 19:566-75.

Mickan S, Hoffman SJ, Nasmith L.,2010. Collaborative practice in a global health context: common themes from developed and developing countries. J Interprof Care. 24:492-502.

Oxford dictionaries. [cited 25 May 2015]. Available from: http://www.oxforddictionaries.com/defini tion.
Rodger S, Hoffman SJ.,2010. Where in the world is interprofessional education? A global environmental scan. J Interprof Care. 24:479-91.

Sutcliffe KM, Lewton E, Rosenthal MM.,2004. Communication failures: an insidious contributor to medical mishaps. Acad Med. 79:186-94.

Suter E, Arndt J, Arthur N, Parboosingh J, Taylor E, Deutschlander S.,2009. Role understanding and effective communication as core competencies for collaborative practice. Journal of interprofessional care. 23:41-51.

Tong A, Sainsbury P, Craig J.,2007. Consolidated criteria for reporting qualitative research (COREQ): a 32-item checklist for interviews and focus groups. Int J Qual Health Care. 19:349-57.

Weidman JC, Twale DJ, Stein EL.,2001 Socialization of graduate and professional students in higher education: a perilous passage. San Francisco, California: The ASHE-ERIC Higher Education Report.

Whitehead C.,2007. The doctor dilemma in interprofessional education and care: how and why will physicians collaborate? Med Educ. 41:1010-16.

World Health Organization., 2010. Framework for Action on Interprofessional Education and Collaborative Practice. Geneva: WHO 